\title{
ON PARALLELOTOPE CONFIGURATION
}

\author{
Attila Végh ${ }^{1 *}$ \\ ${ }^{1}$ Department of Natural Sciences and Engineering, GAMF Faculty of Engineering and Computer Science, \\ John von Neumann University, Hungary \\ https://doi.org/10.47833/2020.3.CSC.002
}

\section{Keywords:}

parallelotope

configuration

\section{Article history:}

Received 29 Sept 2020

Revised 18 Oct 2020

Accepted 25 Oct 2020

\begin{abstract}
The parallelotope $\mathcal{P}$ is a convex polytope which fills the space facet to facet by its translation copies without intersecting by inner points. A plane configuration is a system of $p$ points and $g$ straight lines arranged in a plane in such a way that every point of the system is incident with a fixed number $\gamma$ of straight lines of the system and every straight line of the system is incident with a fixed number $\pi$ of points of the system. In this paper we examine the connection of 3-dimensional parallelotopes and point-line configurations in the plane and we generalize the concept of the configuration to describe all parallelotopes, this way we define the parallelotope configuration ( $p$-configuration).
\end{abstract}

\section{Parallelotopes}

The parallelotope $\mathcal{P}$ is a convex polytope whose translated copies tile the space in a face to face manner. The centers of the parallelotopes form an $n$-dimensional lattice. In the plane there are two types of parallelotopes: parallelograms and centrally symmetric hexagons.

Parallelotopes were characterized by B. A. Venkov[15] and later P. McMullen[10] in the following way

Theorem 1. A polytope $\mathcal{P}$ is a parallelotope if and only if

- $\mathcal{P}$ is centrally symmetric,

- each facet of $\mathcal{P}$ is centrally symmetric, and

- the 2-dimensional orthogonal projection along any $(n-2)$-face of $\mathcal{P}$ is either a parallelogram or a centrally symmetric hexagon.

The edges of the parallelogram and the centrally symmetric hexagon are projections of the facets of the parallelotope $\mathcal{P}$. These facets form a 4 - or a 6 -belt, respectively.

B. A. Venkov introduced the concept of the parallelotope of non-zero width in the direction of a $k$-subspace $X^{k}$. A parallelotope $\mathcal{P}$ has non-zero width along $X^{k}$ if the intersection $\mathcal{P} \cap\left(X^{k}+\mathbf{a}\right)$ is either $k$-dimensional or empty for every translation vector a. Denote by $F(n-1)$ a facet of the parallelotope $\mathcal{P}$ and by $\mathbf{t}$ the lattice vector between the centers of the two nearest parallelotopes $\mathcal{P}$ and $\mathcal{Q}$ where $\mathcal{P}$ and $\mathcal{Q}$ have the common facet $F(n-1)$. This lattice vector is called relevant vector of the facet $F(n-1)$ by [7].

Theorem 2. (B. A. VENKOV [16]) Let $\mathcal{P}$ be an n-dimensional parallelotope of non-zero width along $X^{k}$. Then the projection of $\mathcal{P}$ along $X^{k}$ is a parallelotope (of dimension $n-k$ ) and the lattice vectors $\mathbf{t}_{i}$ related to the facets $F_{i}(n-1)$ which are parallel to $X^{k}$ generate an $(n-k)$-dimensional lattice spanning a space $X^{n-k}$ which is transversal to $X^{k}$.

${ }^{*}$ Corresponding author. Tel. +3676516438

E-mail address: vegh.attila@gamf.uni-neumann.hu 
By this theorem for $k=n-2$ relevant vectors of a 4- or 6-belt span a 2-dimensional lattice, thus these relevant vectors are in a plane.

Consider the parallelotopes $\mathcal{P}$ and $\mathcal{Q}$ of dimension $n$. For $k=1$ denote by $S(\mathbf{z})$ the segment $X^{k}$ of the direction $\mathbf{z}$ and of the length $z$. If there exists a direction $\mathbf{z}$ for which $\mathcal{P} \oplus S(\mathbf{z})=\mathcal{Q}$, where $\oplus$ denotes the Minkowski sum, then $\mathcal{P}$ is called the contraction of $\mathcal{Q}$ and $\mathcal{Q}$ is the extraction of $\mathcal{P}$.

Two parallelotopes in the plane were well-known already in the antiquity: the centrally symmetric hexagon (primitive) and the parallelogram (not primitive). E.S. FEDOROV in [5] described the 5 combinatorically different parallelotopes in 3-dimension among which the truncated octahedron is primitive and the others, namely the elongated octahedron, the rhombic dodecahedron, the hexagonal prism and the cube are not primitive. The following picture shows these 5 parallelotopes and their order indicates the way how you can get one from the other by extraction.
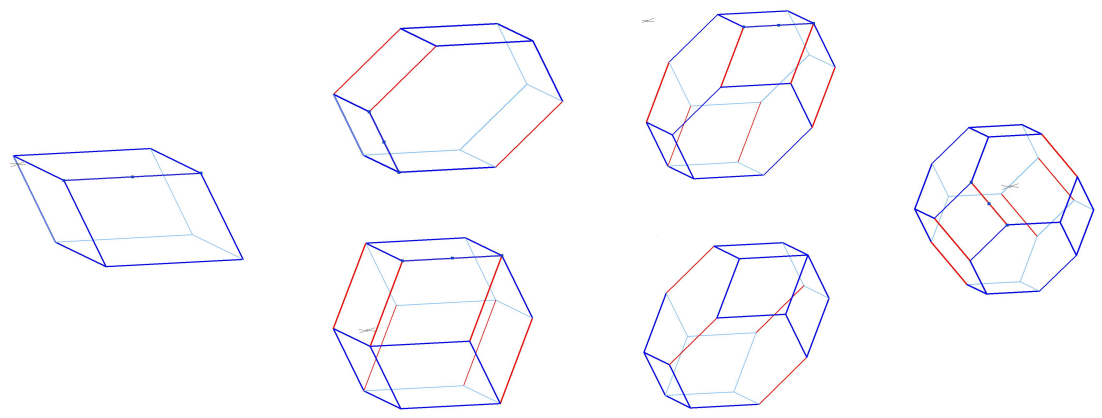

Figure 1. 3-dimensional parallelotopes

B.N. DELONE [1] found 51 different types of the 4-dimensional parallelotopes. M.I. SHTOGRIN gave the missing 52nd in [14]. 17 of these are zonotopes, the other 35 are the regular 24-cell and its Minkowski sum with some zonotopes. Three out of these types are primitive. S.S. RYSHKOV and E.P. BARANOWSKII [13] found 221 primitive 5-dimensional parallelotopes. One more was given by P. ENGEL and V. GRISHUKHIN [4]. P. ENGEL in [2] and [3] gave 179372 combinatorically different types of 5 -dimensional parallelotopes.

In this paper we focus on 3-dimensional parallelotopes.

\section{Configurations}

A configuration is a system of $p$ points and $g$ straight lines arranged in a plane in such a way that every point of the system is incident with a fixed number $\gamma$ of straight lines and every straight line of the system is incident with a fixed number $\pi$ of points. Notation: $\left(p_{\gamma}, g_{\pi}\right)$.

The following relation must be true for every configuration:

$$
p \cdot \gamma=g \cdot \pi \text {. }
$$

The configurations in which the number of points is equal to the number of lines, i.e. for which $p=$ $g$ and consequently $\gamma=\pi$ are called symmetric or balanced configurations. For such a configuration we use the notation $p_{\gamma}$.

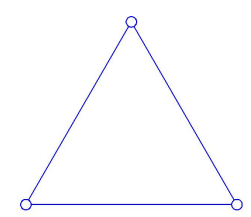

$\left(3_{2}\right)$ symmetric

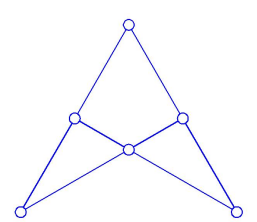

$\left(6_{2}, 4_{3}\right)$ nonsymmetric

Figure 2. Configurations in the plane 
The concept of the configuration may be generalized to higher dimensions, for instance to points and lines in the space. Point-line configurations can be realized in $n$-dimensional euclidean or projective space. For example the Desargues configuration have 10 points and 10 lines, every point lies on 3 lines and every lines contains 3 points, consequently this is a symmetric configuration $\left(10_{3}\right)$. The Reye configuration $\left(12_{4}, 16_{3}\right)$ is nonsymmetric.

Similarly, one can consider configurations of points and planes. In the Reye configuration there are 12 points and 12 planes each point of the configuration belongs to 6 planes, each plane contains 6 points, therefore as point-plane configuration $\left(12_{6}\right)$ is symmetric.

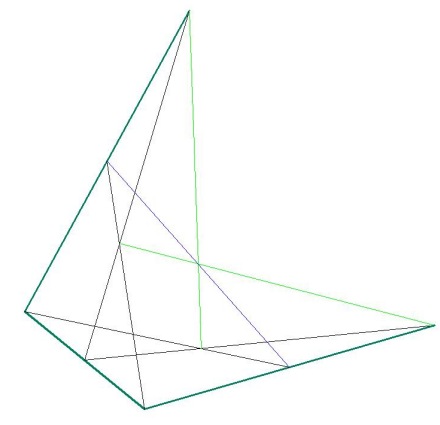

Desargues configuration

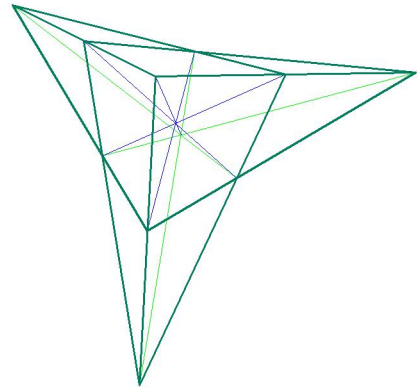

Reye configuration

Figure 3. Configurations in space

Henceforth we are concerned with the connection of point-line configurations and parallelotopes.

\section{Parallelotope configurations}

Every $n$-polytope has a dual structure. It can be obtained by interchanging its vertices and facets, edges and $(n-2)$-dimensional faces, and so on, generally interchanging its $(j-1)$-dimensional elements and $(n-j)$-dimensional elements, preserving incidence between elements. The dual polytope of the polytope $P$ is denoted by $P^{*}$. We use duality for parallelotopes. Facets of a parallelotope $\mathcal{P}$ are centrally symmetric, thus we can define vertices of dual polytope $P^{*}$ by centers of facets of the parallelotope $\mathcal{P}$. For example consider the blue truncated octahedron in the picture 4 . The dual polytope is the red polytope, which is not a parallelotope.

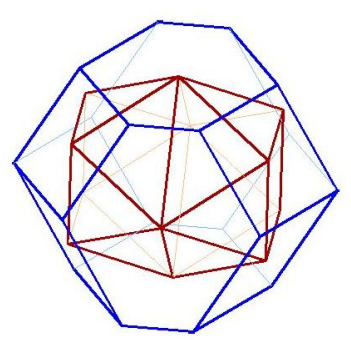

Figure 4. Duality

Consider the dual polytope $\mathcal{P}^{*}$ of a parallelotope $\mathcal{P}$. The parallelotope configuration or $\mathrm{p}$ configuration is a system of lines and points projected vertices and edges of the polytope $\mathcal{P}^{*}$ from the center of the polytope $\mathcal{P}^{*}$ to an $(n-1)$-dimensional hyperplane, which is parallel to a hyperplane containing the center of the parallelotope $\mathcal{P}$ and not containing any vertex of the dual polytope $\mathcal{P}^{*} . \ln$ this manner every parallelotope $\mathcal{P}$ determines a $p$-configuration denoted by $\Pi$. A line of a $p$-configuration $\Pi$ is called $p$-line. An example is presented on figure 5. 


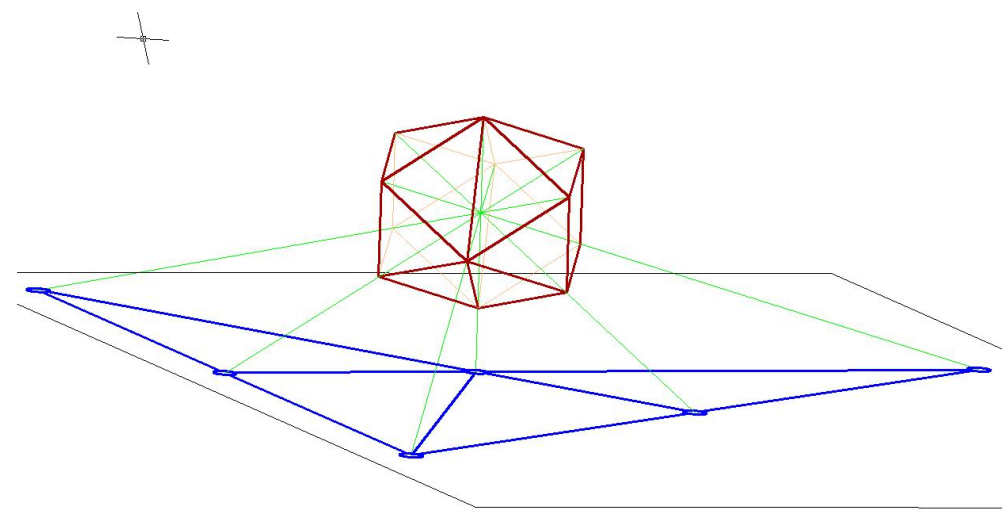

Figure 5. p-configuration

The notation of the p-configuration is

$$
\left(\left(p_{1}\right)_{\gamma_{1}},\left(p_{2}\right)_{\gamma_{2}}, \ldots,\left(p_{k}\right)_{\gamma_{k}} ;\left(\left(g_{1}\right)_{\pi_{1}},\left(g_{2}\right)_{\pi_{2}}, \ldots,\left(g_{l}\right)_{\pi_{l}}\right),\right.
$$

where $p_{i}$ is the number of points which belong to $\gamma_{i}$ lines and $g_{i}$ is the number of lines which contain $\pi_{i}$ points. The sum of $p_{i}$ is equal to the number of all points of the p-configuration and the sum of $g_{i}$ is equal to the number of all lines of the p-configuration. Figure 6 shows $p$-configurations in the plane.
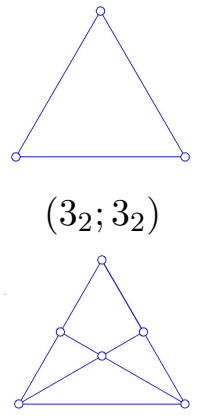

$\left(2_{3}, 4_{2} ; 4_{3}, 1_{2}\right)$

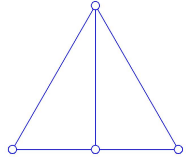

$\left(1_{3}, 3_{2} ; 1_{3}, 3_{2}\right)$

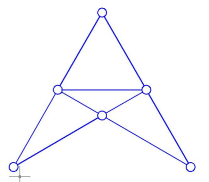

$\left(2_{3}, 4_{2} ; 4_{3}, 1_{2}\right)$

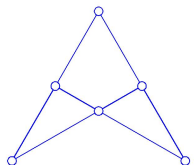

$\left(6_{2} ; 4_{3}\right)$

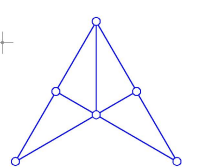

$\left(2_{3}, 4_{2} ; 4_{3}, 1_{2}\right)$

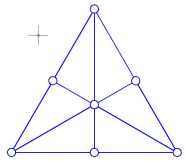

$\left(4_{3}, 3_{2} ; 6_{3}\right)$

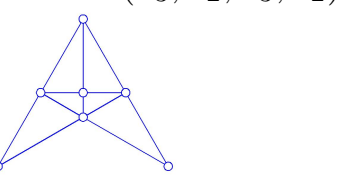

$\left(4_{3}, 3_{2} ; 6_{3}\right)$

Figure 6. p-configuration in the plane

The geometric realizations of the p-configuration $\left(2_{3}, 4_{2} ; 4_{3}, 1_{2}\right)$ and $\left(4_{3}, 3_{2} ; 6_{3}\right)$ are different, but there is a projective transformation between the two geometric realizations.

\section{Properties of the p-configuration}

In this section we examine the properties of the p-configuration.

Theorem 3. Every $p$-line contains two or three points of the p-configuration $\Pi$. Every point of the $p$-configuration $\Pi$ belongs to at least $(n-1)$ straight lines, these lines generate a $(n-1)$-dimensional hyperplane. 
Proof. The parallelotope $\mathcal{P}$ contains 4 - or 6 -belt by theorem 1 , thus the dual polytope $\mathcal{P}^{*}$ has 4 or 6 vertices. On the other hand the parallelotope $\mathcal{P}$ is centrally symmetric, and so is the dual polytope $\mathcal{P}^{*}$. The central projection of 2 opposite vertices is a point on the $(n-1)$-dimensional hyperplane. 4 or 6 vertices of the dual polytope $\mathcal{P}^{*}$ are in a plane by theorem 2 , causing that central projections of these vertices are on a line.

The proof of the second statement. A point of the p-configuration $\Pi$ corresponds to a facet of the parallelotope $\mathcal{P}$ and a line of the $p$-configuration $\Pi$ corresponds to a belt of the parallelotope $\mathcal{P}$. A facet $F_{1}$ of the parallelotope $\mathcal{P}$ is centrally symmetric, so the centrally symmetric image of a $(n-2)$-dimensional face $G_{1}$ of the parallelotope $\mathcal{P}$ is an other $(n-2)$-dimensional face $G_{2}$ of the parallelotope $\mathcal{P}$. The face $G_{2}$ contains an other facet $F_{2}$, this facet is centrally symmetric as well. The face $G_{3}$ is a centrally symmetric image of the face $G_{2}$ to the center of the facet $F_{2}$. Repeating this procedure after 4 or 6 steps we get back the face $G_{1}$ by teorem 1, in this way a $(n-2)$-dimensional face $G_{1}$ of the parallelotope $\mathcal{P}$ determines a belt of the parallelotope $\mathcal{P}$. There are at least $2 *(n-1)$ opposite $(n-2)$-dimensional faces on a facet $F_{i}$ since it is a $(n-1)$-dimensional centrally symmetric polytope. On the hyperplane of the facet $F_{i}$ the normal vectors of the $(n-2)$-dimensional faces $G_{i}$ generate a $(n-1)$-dimensional hyperplane.

So facet $F_{i}$ contains $(n-1)$ different belts and consequently a point of the p-configuration $\Pi$ belongs to at least $(n-1) \mathrm{p}$-lines and these lines generate $\mathrm{a}(n-1)$-dimensional hyperplane.

Definition 1. The shadow boundary of a parallelotope $\mathcal{P}$ in the direction $\mathrm{z}$ consists of all boundary points $\mathbf{x}$ of $\mathcal{P}$ for which the line $\{\mathbf{x}+\lambda \mathbf{z} \mid \lambda \in \mathbb{R}\}$ is a support line of $\mathcal{P}$. (There is no point of the line $\{\mathbf{x}+\lambda \mathbf{z} \mid \lambda \in \mathbb{R}\}$ belonging to the interior of $\mathcal{P})$. It is denoted by $\operatorname{sh}_{\mathbf{z}}(\mathcal{P})$.

It is well known that the shadow boundary of a convex polytope is the union of its several $(n-1)$ and $(n-2)$-dimensional closed faces [9].

V. GRISHUKHIN [6] defined the z-cap of a parallelotope P. A. MAGAZINOV and Á.G. HORVÁtH used this concept in [11], [7].

Definition 2. Let $\mathcal{P}$ be an $n$-dimensional parallelotope and the vector $\mathrm{z}$ be a free vector. The $\mathrm{z}$-cap $\operatorname{Cap}_{\mathbf{z}}(\mathcal{P})$ of a parallelotope $\mathcal{P}$ consists of all the facets $F$ of the parallelotope $\mathcal{P}$ which satisfy the condition

$$
\mathbf{z} \cdot \mathbf{n}(F)<0,
$$

where $\mathbf{n}(F)$ is the normal vector of the facet $F$.

Theorem 4. Both endpoints of every segments of the $p$-configuration $\Pi$ are boundary points of the p-configuration $\Pi$.

Proof. Consider a direction $\mathbf{z}$ for which the shadow boundary $\operatorname{sh}_{\mathbf{z}}(\mathcal{P})$ is the union of only $(n-2)$ dimensional faces. It can easily be seen that projections of center points of facets $F_{i}$ of the $\operatorname{Cap}_{\mathbf{z}}(\mathcal{P})$ for which $\operatorname{sh}_{\mathbf{z}}(\mathcal{P}) \cap \operatorname{Cap}_{\mathbf{z}}(\mathcal{P})$ is a $(n-2)$-dimensional face (that is facets $F_{i}$ adjoin to shadow boundary) are the boundary points of a $\mathrm{p}$-configuration $\Pi$.

Because of central symmetry every belt crosses the shadow boundary $\operatorname{sh}_{\mathbf{z}}(\mathcal{P})$ in two $(n-2)$ dimensional faces. So two facets $F_{1}$ and $F_{2}$ of a belt are on a $\operatorname{Cap}_{\mathbf{z}}(\mathcal{P})$ for which $\operatorname{sh}_{\mathbf{z}}(\mathcal{P}) \cap F_{1}$ and $s h_{\mathbf{z}}(\mathcal{P}) \cap F_{2}$ are $(n-2)$-dimensional faces.

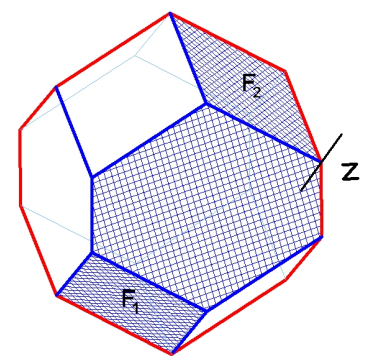

Figure 7. Endpoints of segments 
In this way both endpoints of segments corresponding to this belt are boundary points of a $p$ configuration $\Pi$.

Definition 3. Let $K$ be a $(n-k)$-dimensional face of the parallelotope $\mathcal{P}$. Consider a $k$-dimensional hyperplane $H$ that intersects the face $K$ transversally. In a small neighborhood of the face $K$ the section of the parallelotope tiling by the hyperplane $H$ is called a fan of the face $K$ and denoted by $\operatorname{Fan}(K)$.

B.N. DELONE [1] determined five possible combinatorial types of 3-dimensional fans which can be seen in figure 8 .

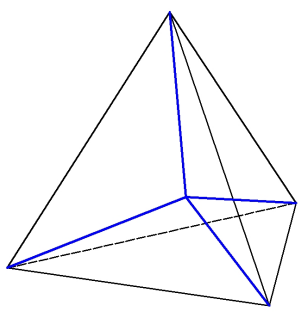

I.

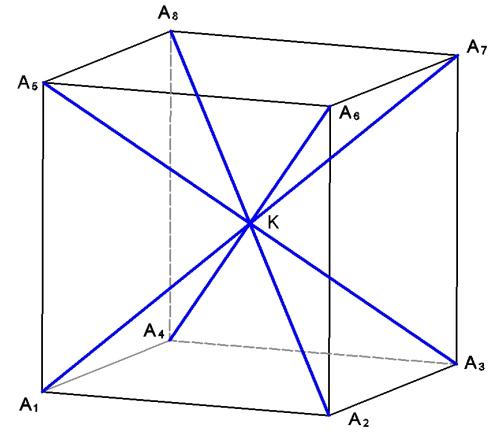

IV.

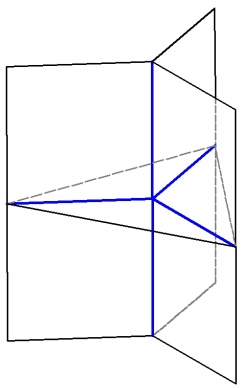

II.

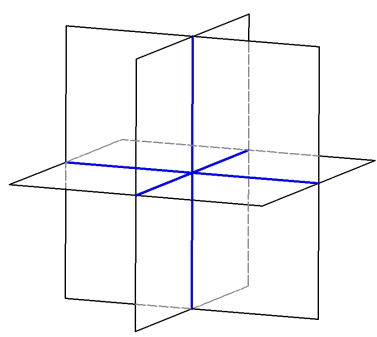

III.

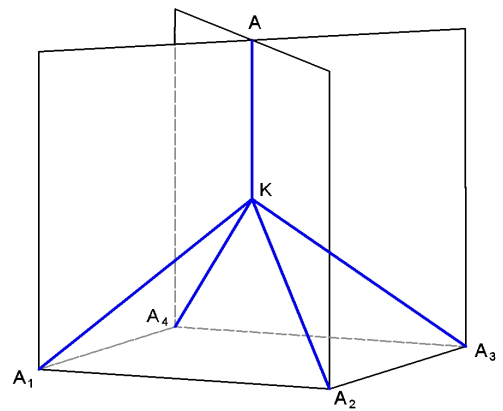

V.

Figure 8. 3-dimensional fans

Lemma 1. Let $K$ be a $(n-3)$-dimensional face and $G_{i}$ be a $(n-2)$-dimensional face and $F_{i}$ be a facet of the parallelotope $\mathcal{P}$. Denote by $B\left(G_{i}\right)$ the set of facets $F_{i}$ which form the belt of the $(n-2)$ dimensional face $G_{i}$. If $K \subseteq G_{1}$ and $K \subseteq G_{2}$ then $B\left(G_{1}\right) \cap B\left(G_{2}\right) \neq \emptyset$.

Proof. In the cases I, II, III if $K \subseteq G_{1}$ and $K \subseteq G_{2}$ then $(n-2)$-dimensional faces $G_{1}$ and $G_{2}$ determine a facet of the parallelotope $\mathcal{P}$, consequently the statement is true.

In the case IV for $G_{1}=\left\langle A_{1}, K\right\rangle$ and $G_{2}=\left\langle A_{2}, K\right\rangle$ the statement is similarly true.

The interesting case is for example $G_{1}=\left\langle A_{1}, K\right\rangle$ and $G_{2}=\left\langle A_{3}, K\right\rangle$. Let $\mathcal{P}_{0}=\left\langle A_{1}, A_{2}, A_{3}, A_{4}, K\right\rangle$, $\mathcal{P}_{1}=\left\langle A_{1}, A_{2}, A_{5}, A_{6}, K\right\rangle, \mathcal{P}_{2}=\left\langle A_{2}, A_{3}, A_{6}, A_{7}, K\right\rangle$. In this situation the central symmetric image of the parallelotope $\mathcal{P}_{1}$ to the center $C_{1}$ of the facet $\left\langle A_{1}, A_{2}, K\right\rangle$ is the parallelotope $\mathcal{P}_{0}$. Similarly the central symmetric image of the parallelotope $\mathcal{P}_{2}$ to the center $C_{2}$ of the facet $\left\langle A_{2}, A_{3}, K\right\rangle$ is the parallelotope $\mathcal{P}_{0}$.

Facets $F_{1}=\left\langle A_{1}, A_{5}, K\right\rangle$ and $F_{2}=\left\langle A_{3}, A_{7}, K\right\rangle$ belong to a common $(n-1)$-dimnesional hyperplane $H$. The central symmetric image of the facet $F_{1}$ to the center $C_{1}$ is a facet $F_{1}^{\prime}$ of the parallelotope $\mathcal{P}_{0}$, which is parallel to the facet $F_{1}$. The central symmetric image of the facet $F_{2}$ to the center $C_{2}$ is also a facet $F_{2}^{\prime}$ of the parallelotope $\mathcal{P}_{0}$, which is parallel to the facet $F_{2}$, consequently $F_{1}^{\prime} \| F_{2}^{\prime}$. Because facets $F_{1}^{\prime}$ and $F_{2}^{\prime}$ are in the same halfspace of the hyperplane $H$, so $F_{1}^{\prime}=F_{2}^{\prime}=F$. On the one hand $G_{1} \subseteq F_{1}$ and $G_{2} \subseteq F_{2}$ therefore $G_{1}^{\prime} \subseteq F$ and $G_{2}^{\prime} \subseteq F$ accordingly $\left\langle G_{1}^{\prime}, G_{2}^{\prime}\right\rangle=F$. On the other hand $B\left(G_{1}\right)=B\left(G_{1}^{\prime}\right)$ and $B\left(G_{2}\right)=B\left(G_{2}^{\prime}\right)$ thus $F \in B\left(G_{1}^{\prime}\right)$ and $F \in B\left(G_{2}^{\prime}\right)$, consequently $F \in\left(B\left(G_{1}\right) \cap B\left(G_{2}\right)\right)$. 
In the case $\mathrm{V}$ choosing $A=A_{5}=A_{6}=A_{7}$ the proof is similar.

Theorem 5. The intersection of two uniplanar $p$-line is a point of the $p$-configuration $\Pi$.

Proof. A facet of the parallelotope $\mathcal{P}$ corresponds to a point of the p-configuration $\Pi$. An $(n-2)$ dimensional face (belt) of the parallelotope $\mathcal{P}$ corresponds to a line of the p-configuration $\Pi$. An $(n-3)$-dimensional face of the parallelotope $\mathcal{P}$ corresponds to a plane of the p-configuration $\Pi$. The duality and the projection preserve the relation $\subseteq$. So if a plane contains two $p$-lines of the $p$ configuration $\Pi$, then an $(n-3)$-dimensional face of the parallelotope $\mathcal{P}$ contains two corresponding $(n-2)$-dimensional faces of the parallelotope $\mathcal{P}$. By lemma 1 the intersection of two $p$-lines corresponding to two $(n-2)$-dimensional faces of the parallelotope $\mathcal{P}$ is a point of the $p$-configuration $\Pi$ which corresponds to the facet of the parallelotope $\mathcal{P}$.

Theorem 6. The convex hull of points of a p-configuration $\Pi$ is a triangle in the plane.

Proof. In the plane the statement follows from theorem 5.

Generally, we form the following conjecture.

Conjecture 1. The convex hull of points of a $p$-configuration is a $(n-1)$-dimensional simplex.

\section{Acknowledgement}

This research is supported by EFOP-3.6.1-16-2016-00006 "The development and enhancement of the research potential at John von Neumann University" project. The Project is supported by the Hungarian Government and co-financed by the European Social Fund.

\section{References}

[1] B.N. Delone, Sur la partition reguliere de l'espace a 4-dimensions, Izv. Akad. Nauk SSSR Otdel. Fiz.-Mat. Nauk 7 (1929) 79-110, 147-164. JFM 56.1120.02

[2] P. Engel, Investigations of parallelohedra in $\mathbf{R}^{d}$, in: Voronoi's impact on Modern Science, P. Engel and H. Syta (eds), Institute of Mathematics, vol.2,Kyiv 1998, 22-60. Zbl 0928.00007

[3] P. Engel, The contraction types of parallelohedra in $E^{5}$, Acta Cryst. Sect. A 56 (2000) 491-496. https://doi.org/10.1107/s0108767300007145

[4] P.Engel, V. Grishukhin, There are exactly 222 L-types of primitive 5-dimensional parallelotopes, Eur. J. Comb. 23, No. 3, 275-279 (2002) https://doi.org/10.1006/eujc.2001.0551

[5] Fedorov, E. S., Elements of the study of figures, Zap. Miner. Obsc. Vol. 21. (1885), 1-279 pp.

[6] V. Grishukhin, Parallelotopes of non-zero width, Sb. Math., 2004, 195 (5), 669-686. https://doi.org/10.1070/sm2004v195n05abeh000821

[7] Á.G.Horváth, On the connection between the projection and the extension of a parallelotope, Monatsh. Math. 150, (2007), 211-216. https://doi.org/10.1007/s00605-005-0413-1

[8] Hilbert, David; Cohn-Vossen, Stephan, Geometry and the Imagination, Chelsea, 1952

[9] H. Martini, Shadow-boundaries of convex bodies, Discrete Math. 155 (1996), 161-172. https://doi.org/10.1016/0012-365x(94)00380-2

[10] P. McMullen, Convex bodies which tile space by translation, Mathematica 27, (1980), 113-121. https://doi.org/10.1007/978-1-4612-5648-9-6

[11] A. Magazinov, Voronoi's conjecture for extensions of Voronoi parallelohedra, Russ. Mat. Surv.69(4) (2014) 763, https://doi.org/10.1070/RM2014v069n04ABEH004914, Zbl 1314.52013 
[12] Reye, Th., Das Problem der Configurationen, Acta Math. Vol. 1. (1882), 93-96 pp. https://doi.org/10.1007/bf02592127

[13] S.S Ryshkov, E.P. Baranowskii, C-types of $n$-dimensional lattices and 5-dimensional primitive parallelohedra (with application to the theory of coverings) Trudy Math. Institute Steklova 137 (1976) (in Russian), translated in Proc. Steklov Inst. Math. No. 4 (1978) Zbl 0419.10031

[14] M.I. Shtogrin, Regular Dirichlet-Voronoi partitions for the second triclinic group, Proc. Stekl. Inst. Math. 123 (1973) 1-127. Zbl 0306.50004

[15] B.A. Venkov, On a class of Euclidean polytopes, Vestnik Leningradskogo Univ. 9,(1954), 11-31. (in Russian) Zbl 0056.14103

[16] B. A. Venkov, On projecting of parallelohedra, Mat. Sbornik 49,(1959), 207-224. (in Russian) Zbl 0087.04201 